\title{
Several types of one-sided partial orders in rings
}

\author{
Huihui Zhu* Pedro Patrício ${ }^{*}$
}

\begin{abstract}
In this paper, we investigate one-sided sharp partial orders and one-sided core and dual core partial orders in rings. Moreover, their characterizations and relations with other partial orders are given.
\end{abstract}

Keywords: group inverses, Moore-Penrose inverses, core inverses, one-sided sharp partial orders, one-sided core partial orders

AMS: 15A09, 06A06, 16W10

\section{Introduction}

There exist specific partial orders in the mathematical literature, such as star partial orders [3], minus partial orders [4], sharp partial orders [7], core and dual core partial orders [2], diamond partial orders [5] and so on. Recently, Marovt [6] and Mosić [8] introduced onesided sharp partial orders and one-sided core partial orders in rings, respectively. Moreover, some properties of them were investigated.

In this article, we further study properties and characterizations of one-sided sharp partial orders and one-sided core and dual core partial orders in rings. The paper is organized as follows. In Section 2, notations and definitions are presented and several known results of generalized inverses and partial orders are given. In Section 3, one condition of Marovt's one-sided sharp partial orders is weakened. Also, we give the existence criterion such that $b$ is group invertible if $a$ is below $b$ with respect to left sharp partial order. In Section 4, we present several characterizations of one-sided core partial orders and their relations with other types of partial orders.

\footnotetext{
${ }^{*}$ School of Mathematics, Hefei University of Technology, Hefei 230009, China. hhzhu@hfut.edu.cn

${ }^{\dagger}$ CMAT-Centro de Matemática and Departamento de Matemática, Universidade do Minho, Braga 4710-057, Portugal. pedro@math.uminho.pt
} 


\section{Preliminaries}

Assume that $R$ is a ring with unity 1 . An element $a \in R$ is (von Neumann) regular if there exists an element $x \in R$ such that $a=a x a$. Such an $x$ is called an inner inverse of $a$, and is denoted by $a^{-}$. The symbol $a\{1\}$ denotes the set of all inner inverses of $a$.

We call $a \in R$ to be group invertible if there exists an element $b \in R$ such that

(i) $a b=b a$,

(ii) $b a b=b$,

(iii) $a b a=a$.

A $b$ satisfying the above conditions (i)-(iii) is called a group inverse of $a$. It is unique if it exists, and is denoted by $a^{\#}$. We denote by $R^{\#}$ the set of all group invertible elements in $R$.

In 1987, Mitra [7] introduced the sharp partial order of square matrices over a field. Let $A$ and $B$ be matrices of index 1. $A$ is below $B$ with respect to the sharp partial order (write $A \leqslant^{\sharp} B$ ) if $A^{\#} A=A^{\#} B$ and $A A^{\#}=B A^{\#}$. Recently, Marovt [6] introduced left and right sharp partial orders in a ring $R$, as extensions of the sharp partial order. Given $a \in R$, we define the sets ${ }^{0} a=\{x \in R: x a=0\}$ and $a^{0}=\{x \in R: a x=0\}$. Following [6], let $a, b \in R^{\#}$. Then (i) $a \sharp \leqslant b$ if and only if $a^{\#} a=a^{\#} b$ and ${ }^{0} b \subseteq{ }^{0} a$. (ii) $a \leqslant \sharp b$ if and only if $a a^{\#}=b a^{\#}$ and $b^{0} \subseteq a^{0}$.

A ring $R$ is called a unital $*$-ring if it is a ring with unity 1 and an involution $*$ satisfying $\left(x^{*}\right)^{*}=x,(x y)^{*}=y^{*} x^{*}$ and $(x+y)^{*}=x^{*}+y^{*}$ for all $x, y \in R$. Let $R$ be a unital *ring. We call $a \in R$ to be $\{1,3\}$-invertible if there exists some $x \in R$ satisfying equations $a x a=a$ and $(a x)^{*}=a x$. Such an $x$ is called a $\{1,3\}$-inverse of $a$, and is denoted by $a^{(1,3)}$. An element $a \in R$ is $\{1,4\}$-invertible if there exists some $y \in R$ such that aya $=a$ and $(y a)^{*}=y a$. Such a $y$ is called a $\{1,4\}$-inverse of $a$, and is denoted by $a^{(1,4)}$. By $a\{1,3\}$ and $a\{1,4\}$ we denote the sets of all $\{1,3\}$-inverses and $\{1,4\}$-inverses of $a$, respectively. It is well known that $a \in R$ is Moore-Penrose invertible if and only if it is both $\{1,3\}$-invertible and $\{1,4\}$-invertible. The standard notion of the Moore-Penrose inverse can be found in [9]. The Moore-Penrose inverse of $a \in R$ is unique if it exists, and is denoted by $a^{\dagger}$. More results on Moore-Penrose inverses can be found in $[13,14,15]$.

An element $a$ in a unital $*$-ring $R$ is called core invertible [11] if there exists an $x \in R$ such that $a x a=a, x R=a R$ and $R x=R a^{*}$. Such an $x$ is called the core inverse of $a$. Dually, $a$ is called dual core invertible if there exists $y \in R$ such that aya $=a, R y=R a$ and $y R=a^{*} R$. Such a $y$ is called the dual core inverse of $a$. The core inverse and the dual core inverse of $a$ are unique, if they exist, and are denoted by $a^{\oplus}$ and $a_{\oplus}$, respectively. As 
usual, by $R^{\dagger}, R^{(1,3)}, R^{(1,4)}, R^{\oplus}$ and $R_{\circledast}$ we denote the sets of all Moore-Penrose invertible, $\{1,3\}$-invertible, $\{1,4\}$-invertible, core invertible and dual core invertible elements in $R$, respectively. It is known ([12]) that $a \in R^{\oplus}$ if and only if $a \in R^{\#} \cap R^{(1,3)}$, and that $a \in R_{\circledast}$ if and only if $a \in R^{\#} \cap R^{(1,4)}$. In this case, $a^{\boxplus}=a^{\#} a a^{(1,3)}$ and $a_{\circledast}=a^{(1,4)} a a^{\#}$. More properties about core inverses and dual core inverses can be seen [16].

Using core and dual core inverses, the core and dual core partial orders were given in [2]. Given core invertible complex matrices $A, B, A$ is below $B$ with respect to the core partial order (denoted by $A \leqslant B$ ) if $A^{\oplus} A=A^{\oplus} B$ and $A A^{\oplus}=B A^{\oplus}$. If $A$ and $B$ are dual core invertible, $A$ is below $B$ with respect to the dual core partial order (denoted by $A \leqslant \oplus B)$ if $A_{\oplus} A=A_{\oplus} A$ and $A A_{\circledast}=B A_{\oplus}$. Later, Mosić [8] extended core and dual core partial orders to one-sided core and dual core partial orders in rings. Indeed, given $a \in R^{\boxplus}$ then

(i) $a$ is below $b$ under the left core partial order, denoted by $a^{l, \oplus} \leqslant b$, if $a^{\oplus} a=a^{\oplus} b$ and $a R \subseteq b R$,

(ii) $a$ is below $b$ under the right core partial order, denoted by $a \leqslant r, \oplus b$, if $a a^{\oplus}=b a^{\oplus}$ and $R a \subseteq R b$.

Given $a \in R_{\circledast}$ then

(iii) $a$ is below $b$ under the left dual core partial order, denoted by $a_{l, \oplus} \leqslant b$, if $a_{\oplus} a=a_{\oplus} b$ and $a R \subseteq b R$,

(iv) $a$ is below $b$ under the right dual core partial order, denoted by $a \leqslant_{r, \oplus} b$, if $a a_{\circledast}=b a_{\circledast}$ and $R a \subseteq R b$.

\section{One-sided sharp partial orders}

In [6] it is assumed $a, b \in R^{\#}$ in when considering one-sided sharp partial orders, and their properties and characterizations are presented. Inspired by [6], we further study onesided sharp partial orders only by assuming $a \in R^{\#}$, and give an existence criterion of $b^{\#}$ provided that $a \sharp \leqslant b$.

Definition 3.1. Let $a, b \in R$ with $a \in R^{\#}$. We say that $a$ is below $b$ under left sharp partial order and write $a \sharp \leqslant b$ if $a^{\#} a=a^{\#} b$ and $a R \subseteq b R$.

The relation defined in Definition 3.1 above is a partial order. Clearly, $\sharp \leqslant$ is reflexive. Let $a, b \in R$ with $a \sharp \leqslant b$ and $b \sharp \leqslant a$. Then $b R \subseteq a R$ and hence $b=a a^{\#} b$. From $a^{\#} a=a^{\#} b$, we have $a=a a^{\#} a=a a^{\#} b=b$.

For the proof of transitivity, let $a \sharp \leqslant b$ and $b \sharp \leqslant c$. Then we have $a^{\#} a=a^{\#} b$ and $b^{\#} b=b^{\#} c$. Hence, $a^{\#} a=a^{\#} b=\left(a^{\#}\right)^{2} a b b^{\#} b=\left(a^{\#}\right)^{2}\left(a b b^{\#}\right) c=\left(a^{\#}\right)^{2} a c=a^{\#} c$ by the 
condition (iii) $a=a b^{\#} b$ in Proposition 3.3 below.

Remark 3.2. Definition 3.1 above differs from Marovt's left sharp partial order in $R$. However, the difference vanishes when $b \in R^{\#}$. Indeed, the implication $R a \subseteq R b \Rightarrow b^{0} \subseteq{ }^{0} a$ is trivial. If $b \in R^{\#}$, then $b^{0} \subseteq{ }^{0} a$ implies $R a \subseteq R b$ by $b\left(1-b b^{\#}\right)=0$.

Proposition 3.3. Let $a, b \in R$. Then

(i) If $a \in R^{\#}$ with $a^{\#} a=a^{\#} b$ and $b R \subseteq a R$, then $a=b$.

(ii) If $b \in R^{\#}$ with $a R \subseteq b R$, then $a=b b^{\#} a$.

(iii) If $a \sharp \leqslant b$ and $b \in R^{\#}$, then $a=a b b^{\#}$ and $a a^{\#}=a b^{\#}$.

Proof. (i) Note that $b R \subseteq a R$ ensures that $b=a x$ for some $x \in R$. Then $b=a a^{\#} a x=$ $a a^{\#} b$, which combines with $a=a a^{\#} a=a a^{\#} b$ giving $a=b$.

(ii) As $a R \subseteq b R$, there exists some $y \in R$ such that $a=b y=b b^{\#} b y=b b^{\#} a$ since $b \in R^{\#}$.

(iii) Since $a \sharp \leqslant b$, i.e., $a^{\#} a=a^{\#} b$ and $a R \subseteq b R$, it follows $a=a a^{\#} b$, and consequently $R a \subseteq R b$. This implies $a=a b b^{\#}$ since $b \in R^{\#}$. Hence, $a b^{\#}=a a^{\#} a b^{\#}=a a^{\#} b b^{\#}=$ $a^{\#} a b b^{\#}=a^{\#} a=a a^{\#}$.

It follows from Proposition 3.3 (iii) above that if $a, b \in R^{\#}$ and $a \sharp \leqslant b$, then $a^{\#}=$ $a a^{\#} b^{\#}=a b^{\#} a^{\#}$.

We next present an existence criterion of $b \in R^{\#}$, provided that $a \in R^{\#}$ and $a \sharp \leqslant b$. First, an auxiliary lemma is given.

Lemma 3.4. Let $a, b \in R^{\#}$ and $a \sharp \leqslant b$. Then $a^{\#}=a a^{\#} b^{\#}=b b^{\#} a^{\#}$.

Proof. The proof is essentially given in [6, Lemma 2.6 and Theorem 2].

Theorem 3.5. Let $a, b \in R$ with $a \in R^{\#}$ such that $a \sharp \leqslant b$. Then $b \in R^{\#}$ if and only if $c=b-b a a^{\#} \in R^{\#}$. In this case, $b^{\#}=a^{\#}+c^{\#}-c^{\#} b a^{\#}$ and $c^{\#}=b^{\#}-b^{\#} a a^{\#}$.

Proof. Suppose $b \in R^{\#}$. To show that $c=b-b a a^{\#} \in R^{\#}$, it is sufficient to find an element $x \in R$ satisfying (i) $c x=x c$, (ii) $c x c=c$ and (iii) $x c x=x$. Indeed, $x=b^{\#}-b^{\#} a a^{\#}$ is the group inverse of $c$.

(i) Notice from Lemma 3.4 that $b b^{\#} a a^{\#}=a a^{\#}$ and $a^{\#}=a a^{\#} b^{\#}$. Then, we have $c x=\left(b-b a a^{\#}\right)\left(b^{\#}-b^{\#} a a^{\#}\right)=b b^{\#}-a a^{\#}$. Again, it follows from $a^{\#} a=a^{\#} b$ that $x c=\left(b^{\#}-b^{\#} a a^{\#}\right)\left(b-b a a^{\#}\right)=b b^{\#}-a a^{\#}$. Thus, $c x=x c$.

(ii) $c x c=\left(b b^{\#}-a a^{\#}\right)\left(b-b a a^{\#}\right)=b-b a a^{\#}-a a^{\#} b+a a^{\#} b a a^{\#}=c-a a^{\#} a+a a^{\#} a a a^{\#}=c$.

(iii) $x c x=\left(b^{\#}-b^{\#} a a^{\#}\right)\left(b b^{\#}-a a^{\#}\right)=b^{\#}-b^{\#} a a^{\#}-b^{\#} a a^{\#} b b^{\#}+b^{\#} a a^{\#}=b^{\#}-$ $b^{\#} a a^{\#} b b^{\#}=b^{\#}-b^{\#} a b^{\#}=b^{\#}-b^{\#} a a^{\#}=x$. 
Therefore, $c=b-b a a^{\#} \in R^{\#}$ and $c^{\#}=b^{\#}-b^{\#} a a^{\#}$.

Conversely, as $c=b-b a a^{\#} \in R^{\#}$, then $c a^{\#}=a^{\#} c=0$ by $a^{\#} a=a^{\#} b$. Also, it follows $c^{\#} a=a c^{\#}=0$ since $c^{\#} a=\left(c^{\#}\right)^{2} c a^{\#} a^{2}$ and $a c^{\#}=a^{2} a^{\#} c\left(c^{\#}\right)^{2}$. Hence, $c c^{\#}=$ $\left(b-b a a^{\#}\right) c^{\#}=b c^{\#}-b a^{\#} a c^{\#}=b c^{\#}$. Let us now show that $y=a^{\#}+c^{\#}-c^{\#} b a^{\#}$ is the group inverse of $b$.

(i) $b y b=b\left(a^{\#}+c^{\#}-c^{\#} b a^{\#}\right) b=b a^{\#} a+b c^{\#}\left(b-b a^{\#} b\right)=b a^{\#} a+b c^{\#} c=b a^{\#} a+c c^{\#} c=$ $b a^{\#} a+c=b$ since $c=b-b a^{\#} a$.

(ii) $y b=\left(a^{\#}+c^{\#}-c^{\#} b a^{\#}\right) b=a^{\#} b+c^{\#} b-c^{\#} b a^{\#} b=a^{\#} a+c^{\#} b-c^{\#} b a^{\#} a=a^{\#} a+$ $c^{\#}\left(b-b a^{\#} a\right)=a^{\#} a+c^{\#} c$. Again, from $a R \subseteq b R$ and $b y b=b$, we conclude that $a=b y a=$ $b a^{\#} a-b c^{\#} b a^{\#} a$, and consequently $a a^{\#}=b a^{\#}-b c^{\#} b a^{\#}$. We get $b y=b a^{\#}+b c^{\#}-b c^{\#} b a^{\#}=$ $a a^{\#}+b c^{\#}=a a^{\#}+c c^{\#}$.

(iii) $y b y=\left(a^{\#}+c^{\#}-c^{\#} b a^{\#}\right)\left(a a^{\#}+c c^{\#}\right)=a^{\#}+c^{\#}-c^{\#} b a^{\#}=y$ since $a^{\#} c=c^{\#} a=0$.

Therefore, $b \in R^{\#}$ and $b^{\#}=a^{\#}+c^{\#}-c^{\#} b a^{\#}$.

Let us recall the fact [10, Proposition 7] that $b \in R^{\#}$ if and only if there exist some $s, t \in R$ such that $b=b^{2} s=t b^{2}$. Moreover, $b^{\#}=t b s$. Hence, there is an alterative proof for $b^{\#}=a^{\#}+c^{\#}-c^{\#} b a^{\#}$ in the "if" part of Theorem 3.5 above. Notice that the conditions $b y b=b$ and $b y=y b$ imply $b=b^{2} y=y b^{2}$, and consequently $b^{\#}=y b y$. A direct calculation gives $b^{\#}=y b y=\left(a^{\#} a+c^{\#} b-c^{\#} b a^{\#} a\right)\left(a^{\#}+c^{\#}-c^{\#} b a^{\#}\right)=a^{\#}+c^{\#}-c^{\#} b a^{\#}$.

Dually, we give the definition of the right sharp partial order.

Definition 3.6. Let $a, b \in R$ with $a \in R^{\#}$. We say that $a$ is below $b$ under the right sharp partial order and write $a \leqslant \sharp b$ if $a a^{\#}=b a^{\#}$ and $R a \subseteq R b$.

The left sharp partial order may not be the right sharp partial order in general. A counterexample can be found in [6, page 8$]$.

Let $a \in R^{\#}$. Then $a$ is below $b$ under the sharp partial order if $a^{\#} a=a^{\#} b$ and $a a^{\#}=b a^{\#}$. We remark that $a$ is below $b$ under the sharp partial order if and only if it is below $b$ under both left and right sharp partial orders.

Marovt [6] has considered relations among the sharp partial order, the left and the right sharp partial orders, under the conditions $a, b \in R^{\#}$. Inspired by [6, Theorem 7], we present another characterization of $a \leqslant \sharp$, provided that $a \in R^{\#}$.

Theorem 3.7. Let $a \in R^{\#}$. Then the following conditions are equivalent:

(i) $a \leqslant \sharp$.

(ii) $a \sharp \leqslant b$ and $a b=b a$.

(iii) $a \leqslant \sharp b$ and $a b=b a$. 
Proof. (i) $\Rightarrow$ (ii) As $a \leqslant \sharp b$, then $a^{\#} b=a^{\#} a=a a^{\#}=b a^{\#}$. By the double commutativity of the group inverse, we get $\left(a^{\#}\right)^{\#} b=b\left(a^{\#}\right)^{\#}$, i.e., $a b=b a$. Notice that $a=a a^{\#} a=b a^{\#} a$. Then $a R=b a^{\#} a R \subseteq b R$, which together with $a^{\#} a=a^{\#} b$ implies $a \sharp \leqslant b$.

(ii) $\Rightarrow$ (iii) From $a b=b a$, it follows $a^{\#} b=b a^{\#}$ and hence $a a^{\#}=a^{\#} a=a^{\#} b=b a^{\#}$. Since $a^{\#} a=a^{\#} b$, we obtain $a=a a^{\#} b$ and $R a \subseteq R b$.

(iii) $\Rightarrow$ (i) Note that $a b=b a$ ensures that $a^{\#}$ and $b$ commute. Then the implication is straightforward.

\section{One-sided core and dual core partial orders}

Let us recall the conceptions of left and right star partial orders [1]. Given $a, b \in R$, we say that $a$ is below $b$ under the left star partial order $(a * \leqslant b)$ if $a^{*} a=a^{*} b$ and $a R \subseteq b R$, and that $a$ is below $b$ under the right star partial order $(a \leqslant * b)$ if $a a^{*}=b a^{*}$ and $R a \subseteq R b$. We next consider the relations among one-sided core partial orders, sharp partial orders and star partial orders.

Theorem 4.1. Let $a \in R^{\oplus}$. Then

(i) $a^{l, \oplus} \leqslant b$ if and only if $a * \leqslant b$.

(ii) $a \leqslant r$,® $b$ if and only if $a \leqslant \sharp b$.

Proof. “ $\Rightarrow$ " As $a^{l, \oplus} \leqslant b$, then $a^{\boxplus} a=a^{\oplus b}$ and $a R \subseteq b R$. Multiplying $a^{\oplus} a=a^{\boxplus} b$ by $a$ on the left side gives $a=a a^{\boxplus} b=\left(a a^{\boxplus}\right)^{*} b$, and consequently $a^{*} a=a^{*}\left(a a^{\boxplus}\right)^{*} b=\left(a a^{\boxplus} a\right)^{*} b=a^{*} b$. So $a * \leqslant b$.

" $\Leftarrow$ " Since $a * \leqslant b$, we have $a^{*} a=a^{*} b$ and hence $a=\left(a^{\oplus}\right)^{*} a^{*} a=\left(a^{\oplus}\right)^{*} a^{*} b=a a^{\oplus} b$. Pre-multiplying $a=a a^{\oplus} b$ by $a^{\boxplus}$ concludes $a^{\boxplus} a=a^{\boxplus} b$. It follows from $a R \subseteq b R$ that $a^{l, \oplus} \leqslant b$.

(ii) Suppose $a \leqslant^{r, \oplus b} b$. Then $a a^{\oplus}=b a^{\oplus}$ and $R a \subseteq R b$. Multiplying $a a^{\oplus}=b a^{\oplus}$ by $a a^{\#}$ on the right side gives $a a^{\#}=b a^{\#}$, which together with $R a \subseteq R b$ guarantee $a \leqslant \sharp b$.

Conversely, as $a \leqslant \sharp b$, i.e., $a a^{\#}=b a^{\#}$ and $R a \subseteq R b$, then we get $a a^{\#} a a^{(1,3)}=$ $b a^{\#} a a^{(1,3)}$, that is $a a^{\boxplus}=b a^{\boxplus}$ since $a^{\boxplus}=a^{\#} a a^{(1,3)}$, as required.

Dually, we have the following result.

Theorem 4.2. Let $a \in R_{\circledast}$. Then

(i) $a_{l, \oplus} \leqslant b$ if and only if $a \sharp \leqslant b$.

(ii) $a \leqslant r, \oplus b$ if and only if $a \leqslant * b$. 
In a $*$-ring $R$, the condition $a^{\oplus} a=a^{\oplus} b$ implies $a=a a^{\oplus} b$, and consequently $R a \subseteq R b$. Also, $a a^{\oplus}=b a^{\oplus}$ gives $a R \subseteq b R$. Hence, $a \leqslant \leqslant^{\oplus}$ if and only if $a^{l, \oplus \leqslant b}$ and $a \leqslant r, \oplus b$. We combine Theorem 4.1 and Theorem 4.2 to get the following corollary.

Corollary 4.3. Let $a \in R^{\#} \cap R^{\dagger}$. Then $a \leqslant$ if and only if $a \leqslant \sharp b$ and $a * \leqslant b$.

Recall that the minus partial order is defined in the following way. For $a, b \in R$, where $a$ is regular, we write $a \leqslant^{-} b$ if $a^{-} a=a^{-} b$ and $a a^{-}=b a^{-}$for $a^{-} \in a\{1\}$. A characterization of the minus partial order is that $a \leqslant^{-} b$ if and only if there exists $b^{-} \in b\{1\}$ such that $a=b b^{-} a=a b^{-} b=a b^{-} a$.

We next consider under what conditions one-sided core and dual core partial orders are equivalent to the minus partial order. First, we give the following lemma.

Lemma 4.4. Let $a, b \in R$. Then

(i) If $a, b \in R^{\oplus}$ and $a^{l, \oplus} \leqslant b$, then $a \leqslant-b$.

(ii) If $a, b \in R_{\circledast}$ and $a_{l, \circledast} \leqslant b$, then $a \leqslant-b$.

(iii) If $a, b \in R^{\oplus}$ and $a \leqslant r$,® $b$, then $a \leqslant-b$.

(iv) If $a, b \in R_{\oplus}$ and $a \leqslant r, \oplus b$, then $a \leqslant^{-} b$.

Proof. (i) As $a^{l, \oplus} \leqslant b$, i.e., $a^{\boxplus a} a=a^{\oplus b}$ and $a R \subseteq b R$, then we have $a=a a^{\oplus} a=a a^{\boxplus b}$ and hence $R a \subseteq R b$, which gives $a=x b=x b b^{\oplus b} b a b^{\oplus b}$ for some $x \in R$. Note also that $a R \subseteq b R$ ensures $a=b b^{\oplus a} a$. By a direct calculation, we have $a b^{\oplus} a=a\left(a^{\oplus} a\right) b^{\oplus} a=$ $a\left(a^{\oplus} b\right) b^{\oplus} a=a a^{\oplus} b b^{\oplus} a=a a^{\oplus} a=a$. Therefore, $a=b b^{\oplus} a=a b^{\oplus} b=a b^{\oplus} a$ for $b^{\oplus} \in b\{1\}$. We hence conclude $a \leqslant-b$.

(ii) Given $a_{l, \oplus} \leqslant b$, we get $a=a a_{\oplus} b$, which implies $R a \subseteq R b$. Also, we obtain $a=b b_{\oplus} a=a b_{\oplus} b$ from $a R \subseteq b R$ and $R a \subseteq R b$. As $b_{\oplus}$ is an inner inverse of $a$, indeed, $a b_{\circledast} a=a a_{\circledast} a b_{\circledast} a=a a_{\circledast} b b_{\circledast} a=a a_{\circledast} a=a$, then there exists $b_{\circledast} \in b\{1\}$ such that $a=$ $b b_{\oplus} a=a b_{\oplus} b=a b_{\oplus} a$. Thus, $a \leqslant^{-} b$.

(iii) and (iv) can be obtained similarly.

Theorem 4.5. Let $a, b \in R$. Then we have

(i) If $a, b \in R^{\oplus}$, then $a^{l, \oplus} \leqslant b$ if and only if $a \leqslant^{-} b$ and $R a=R a a^{\boxplus} b$.

(ii) If $a, b \in R_{\oplus}$, then $a_{l, \oplus} \leqslant b$ if and only if $a \leqslant-b$ and $R a=R a b$.

(iii) If $a, b \in R^{\boxplus}$, then $a \leqslant^{r, \oplus b}$ if and only if $a \leqslant^{-} b$ and $a R=b a R$.

(iv) If $a, b \in R_{\oplus}$, then $a \leqslant_{r, \oplus b} b$ if and only if $a \leqslant^{-} b$ and $a R=b a_{\oplus} a R$.

Proof. Suppose $a^{l, \oplus} \leqslant b$. Then, by Lemma 4.4, $a \leqslant^{-} b$. Also, $a^{\oplus} a=a^{\oplus} b$ gives $a=a a^{\oplus b}$. Thus, $R a=R a a^{\oplus b}$. 
Conversely, as $a \leqslant^{-} b$, then $a=a a^{-} b=b a^{-} a$ for some $a^{-} \in a\{1\}$, and consequently $a R=b a^{-} a R \subseteq b R$. Next, we will prove that $a^{\oplus} a=a^{\boxplus b}$. From $a\left(1-a^{-} a\right)=0$ and $R a=R a a^{\boxplus} b$, it follows $a a^{\boxplus} b\left(1-a^{-} a\right)=0$. That is $a a^{\boxplus} b=a a^{\boxplus} b a^{-} a=a a^{\boxplus} a=a$. Thus, $a^{\boxplus} a=a^{\boxplus} a a^{\boxplus} b=a^{\boxplus} b$, as required.

(ii) Suppose $a_{l, \oplus} \leqslant b$. Then $a \leqslant-b$ by Lemma 4.4. We know that $a_{\oplus} a=a_{\oplus} b$ concludes $a=a a_{\circledast} b$, and consequently $a=a a^{\#} b$ since $a a_{\circledast}=a a^{\#}$. Then $R a=R a a^{\#} b=R a b$ by noting $R a=R a a^{\#}$.

For the converse, as $a \leqslant^{-} b$, then $a=a a^{-} b=b a^{-} a$ and $a R \subseteq b R$. Since $R a=R a b$, we get $a b\left(1-a^{-} a\right)=0$. So, $a b=a b a^{-} a=a^{2}$. Pre-multiplying $a b=a^{2}$ by $a^{(1,4)} a^{\#}$ implies $a_{\oplus} a=a_{\oplus} b$.

(iii) and (iv) can be proved by a similar way of (i) and (ii).

It follows from [6, Lemma 3.2] that if $a, b, a-b \in R^{\#}$ then $a \sharp \leqslant b$ if and only if $(b-a) \sharp \leqslant b$. It is natural to ask whether one-sided core partial orders have a similar property, i.e., whether $a^{l, \oplus} \leqslant b$ is equivalent to $(b-a)^{l, \oplus} \leqslant b$. Theorem 4.7 below illustrates the possibility, under certain conditions.

Lemma 4.6. Let $a, b \in R$. Then

(i) $a * \leqslant b$ if and only if $(b-a) * \leqslant b$.

(ii) $a \leqslant * b$ if and only if $(b-a) \leqslant * b$.

Proof. (i) Suppose $a * \leqslant b$. Then $a^{*} a=a^{*} b$ and $a R \subseteq b R$. It follows that $(b-a)^{*} a=0$, and hence $(b-a)^{*}(b-a)=(b-a)^{*} b$. Also, $a R \subseteq b R$ implies $b-a \in b R$, and consequently $(b-a) R \subseteq b R$, as required.

For the converse, note that $(b-a) * \leqslant b$. Then $b-(b-a)=a * \leqslant b$

(ii) can be proved similarly.

Theorem 4.7. Let $a, b-a \in R^{\oplus}$. Then

(i) $a^{l, \oplus} \leqslant b$ if and only if $(b-a)^{l, \oplus} \leqslant b$.

(ii) $a \leqslant r, \oplus b$ if and only if $(b-a) \leqslant r, \oplus b$.

Proof. (i) It follows from Theorem 4.1 and Lemma 4.6 that $a^{l, \oplus \leqslant b}$ if and only if $a * \leqslant b$ if and only if $(b-a) * \leqslant b$. Again, by Theorem 4.1, we know that $(b-a)^{l, \oplus} \leqslant b$ if and only if $(b-a) * \leqslant b$. Hence, $a^{l, \oplus} \leqslant b$ if and only if $(b-a)^{l, \oplus} \leqslant b$.

(ii) can be proved similarly.

Analogously, we obtain similar properties of other one-sided core partial orders. The details are left to the reader. 
It is known that $a \leqslant \leqslant^{\oplus}$ if and only if $a^{l, \oplus} \leqslant b$ and $a \leqslant^{r, \oplus} b$. Applying Theorem 4.7, we get the following result.

Corollary 4.8. Let $a, b-a \in R^{\oplus}$. Then $a \leqslant \leqslant^{\boxplus}$ if and only if $(b-a) \leqslant^{\boxplus} b$.

\section{Ackowledments}

The authors are highly grateful to the referees for their valuable comments and suggestions which greatly improved this paper. This research is supported by the National Natural Science Foundation of China (No. 11801124), the Natural Science Foundation of Anhui Province (No. 1808085QA16), the Fundamental Research Funds for the Central Universities (No. JZ2018HGTB0233) and the Portuguese Funds through FCT- 'Fundação para a Ciência e a Tecnologia', within the project UID-MAT-00013/2013.

\section{References}

[1] J.K. Baksalary, S.K. Mitra, Left-star and right-star partial orderings, Linear Algebra Appl. 149 (1991) 73-89.

[2] O.M. Baksalary, G. Trenkler, Core inverse of matrices, Linear Multilinear Algebra 58 (2010) 681-697.

[3] M.P. Drazin, Natural structures on semigroups with involution, Bull. Amer. Math. Soc. 84 (1978) 139-141.

[4] R.E. Hartwig, How to partially order regular elements, Math. Jpn. 25 (1980) 1-13.

[5] L. Lebtahi, P. Patrício, N. Thome, The diamond partial order in rings, Linear Multilinear Algebra 62 (2014) 386-395.

[6] J. Marovt, One-sided sharp order in rings, J. Algebra. Appl. 15 (2016) 1650161 (10 pages).

[7] S.K. Mitra, On group inverse and the sharp order, Linear Algebra Appl. 92 (1987) $17-37$.

[8] D. Mosić, One-sided core partial orders in a ring with involution, Revista De La Real Academia De Ciencias Exactas Fsicas Y Naturales. serie A. matemticas, 6 (2017) 1-13. 
[9] R. Penrose, A generalized inverse for matrices, Proc. Camb. Phil. Soc. 51 (1955) 406413.

[10] R.E. Hartwig, Block generalized inverses, Arch. Ration. Mech. Anal. 61 (1976) 197251.

[11] D.S. Rakić, N.C. Dinčić, D.S. Djordjević, Group, Moore-Penrose, core and dual core inverse in rings with involution, Linear Algebra Appl. 463 (2014) 115-133.

[12] S.Z. Xu, J.L. Chen, X.X. Zhang, New characterizations for core inverses in rings with involution. Front. Math. China 12 (2017) 231-246.

[13] H.H. Zhu, On DMP inverses and $m$-EP elements in rings, Linear Multilinear Algebra 67 (2019) 756-766.

[14] H.H. Zhu, Several characterizations for generaized inverses in a ring, Linear Multilinear Algebra 66 (2018) 1351-1361.

[15] H.H. Zhu, J.L. Chen, P. Patrício, X. Mary, Centralizer's applications to the inverse along an element, Appl. Math. Comput. 315 (2017) 27-33.

[16] H.H. Zhu, P. Patrício, Characterizations for pseudo core inverses in a ring with involution, Linear Multilinear Algebra 67 (2019) 1109-1120. 